\title{
Spectroscopic Fingerprints of $\mathrm{MgCl}_{2} / \mathrm{TiCl}_{4}$ Nanoclusters Determined by Machine Learning and DFT
}

\author{
Maddalena D'Amore* ${ }^{1}$ Gentoku Takasao, ${ }^{2}$ Hiroki Chikuma, ${ }^{2}$ Toru \\ Wada, ${ }^{2}$ Toshiaki Taniike*, ${ }^{2}$ Fabien Pascale, ${ }^{3}$ and Anna Maria Ferrari ${ }^{1}$ \\ ${ }^{1}$ Dipartimento di Chimica, Università di Torino, \\ Via P. Giuria 5, 10125 Torino, Italy \\ ${ }^{2}$ Graduate School of Advanced Science and Technology, \\ Japan Advanced Institute of Science and Technology, \\ 1-1 Asahidai, Nomi, Ishikawa, 923-1292, Japan \\ ${ }^{3}$ Université de Lorraine - Nancy, CNRS, \\ Laboratoire de Physique et Chimie Théoriques, \\ UMR 7019, Vandoeuvre-les-Nancy, France
}

(Dated: August 19, 2021)

Keywords: polymerization catalysis, machine learning, nanoclusters, DFT, Raman spectrum, IR spectrum 
In this part, we provide further details about the adopted DFT methodology, thermodynamic estimates of adsorption energies through other density functionals and complementary material for the discussion of spectroscopic characterization of $\mathrm{Ti}_{2} \mathrm{Cl}_{8}$ dimer in addition to main text.

\section{A. DFT calculations details}

The thermodynamics of a single site adsorption obtained by means of M06 and PBE0D3 methods is reported for the $\mathrm{TiCl}_{4}$ and $\mathrm{Ti}_{2} \mathrm{Cl}_{8}$ dimer to substrate, for all the elements ( $\mathrm{Mg}$, $\mathrm{Ti}$ and $\mathrm{Cl}$ atoms) the adopted basis set is the split valence triple-zeta basis sets plus polarization (TZVP) of Gaussian type ${ }^{1}$, as indicated in the section Computational Models and Details of main text. Comparison with surface exposing similarly coordinated $\mathrm{Mg}^{2+}$ ions is reported.

In periodic cases the DFT exchange-correlation contribution and its gradient are evaluated by numerical integration over the unit cell volume. The generation of the integration grid points is based on an atomic partition method, originally proposed by Becke $^{2}$, in which the radial and angular points are obtained from Gauss-Legendre quadrature and Lebedev two-dimensional distributions respectively. The choice of a suitable grid is crucial both for numerical accuracy and cost consideration. In this study a pruned grid with 75 radial and 974 angular points has been used.

The truncation criteria of the Coulomb and exchange infinite lattice series are controlled by five thresholds, $\mathrm{T}_{i}$, which have been set to $8\left(\mathrm{~T}_{1}-\mathrm{T}_{4}\right)$ and $20\left(\mathrm{~T}_{5}\right)$. The convergence tolerance on energy for the SCF step has been set to $10^{-8} \mathrm{E}_{h}$ for structural optimizations, and to $10^{-11} \mathrm{E}_{h}$ for frequency calculations. The same methodologies have been employed in the cases of both $\mathrm{TiCl}_{4}$ and $\mathrm{Ti}_{2} \mathrm{Cl}_{8}$ on different sites of interest for a single platelet system.

All common generalized gradient approximation (GGA) DF's adopted in DFT calculations, either pure or hybrid, while effectively accounting for correlation in the range of overlapping densities, fail to describe the long-range electron correlations responsible for van der Waals forces. Including such forces is very important for a correct structural description of system with weakly bound layers but we demonstrated that it becomes mandatory when modeling the adsorption of $\mathrm{TiCl}_{4}$ on $\mathrm{MgCl}_{2}$ surfaces. Hence, we report hereafter the results for dispersion corrected B3LYP-D2 and PBE0-D3 (including three bodies correction) ad- 
sorption energies, together with the outcomes of a non-local meta-GGA by Truhlar relative to plausible precatalytic $\mathrm{Ti}_{x} \mathrm{Cl}_{y}$ species on the nanocrystal. A physisorption process can be assumed in the case of $\mathrm{TiCl}_{4}$ on site E reported in Figure 3 of main text whereas an adsorption of $\mathrm{Ti}_{2} \mathrm{Cl}_{8}$ is observed on the same type sites (see model sketched in panel a of Figure 6), the chemisorption of the monomer $\mathrm{TiCl}_{4}$ occurs on adjacent $\mathrm{B}$ sites of Figure 3 (model sketched in panel d of Figure 4).

Harmonic frequencies, $\omega_{p}$ for surfaces were obtained by diagonalization of the mass-weighted Hessian matrix of second energy derivatives with respect to atomic displacements $u:^{3-7}$

$$
W_{a i, b j}^{\Gamma}=\frac{H_{a i, b j}^{\mathbf{0}}}{\sqrt{M_{a} M_{b}}} \quad \text { with } \quad H_{a i, b j}^{\mathbf{0}}=\left(\frac{\partial^{2} E}{\partial u_{a i}^{\mathbf{0}} \partial u_{b j}^{\mathbf{0}}}\right)
$$

where atoms $a$ and $b$ (with atomic masses $M_{a}$ and $M_{b}$ ) in the reference cell, $\mathbf{0}$, are displaced along the $i$-th and $j$-th Cartesian directions, respectively.

The vibrational analysis allowed us to calculate $\Delta \mathrm{S}_{a d s}$ both at standard conditions $(\mathrm{P}=$ 1 bar, $\mathrm{T}=298 \mathrm{~K}$ ) and at $\mathrm{T}=100 \mathrm{~K}$, therefore together with $\Delta \mathrm{E}_{a d s}$, we evaluated the Gibbs free energy of adsorption, $\Delta \mathrm{G}_{a d s}$, at different temperatures. Infrared intensities are evaluated through Berry phase approach, ${ }^{8}$ which evaluates the atomic Born tensors from the polarizations generated by small atomic displacements (the same as for the second energy derivative).

The relative Raman intensities of the peaks are also computed analytically through a similar scheme. ${ }^{9,10}$

\section{Details of IR and Raman characterization of $\mathrm{Ti}_{2} \mathrm{Cl}_{8}$ dimer}

When we consider the adsorption of a dimer on the cluster, significant differences are observed with reference to the naked nanoplate:

i) the single band at $392 \mathrm{~cm}^{-1}$ related in the naked platelet to $\mathrm{P} \mathrm{Mg}^{2+}$, splits in two bands at 387 and $391 \mathrm{~cm}^{-1}$ referring to the deformation of four-membered Ti-Cl-Ti-Cl ring;

ii) a weak and clearly distinguishable band at $476 \mathrm{~cm}^{-1}$ peeps up;

iii) at lower wavenumbers, namely at 315,323 and $331 \mathrm{~cm}^{-1}$ the bands will undergo a huge reduction in intensity together with a tiny red shift, the involved modes concerning the deformation of $\mathrm{Mg}-\mathrm{Cl}-\mathrm{Mg}$ angles of $\mathrm{Mg}-\mathrm{Cl}-\mathrm{Mg}-\mathrm{Cl}$ underneath rings, since those movements 
are frustrated by the attached dimer.

iv) a small band is also observed in the region $353-369 \mathrm{~cm}^{-1}$ involving more external $\mathrm{Cl}$ atoms anchoring the $\mathrm{Ti}_{2} \mathrm{Cl}_{8}$ dimer to the substrate.

Apart from the low lying bulk modes at 256, 279, 284, $298 \mathrm{~cm}^{-1}$, the Raman feature associated to the adduct, emerges as a triplet of bands at 458, 476, $495 \mathrm{~cm}^{-1}$ where: i) the band at $458 \mathrm{~cm}^{-1}$ mainly arising from the opposite pyramidalization of $\mathrm{Ti}_{1}$ and $\mathrm{Ti}_{2}$ in the cycle Ti-Cl-Ti-Cl corresponding to the normal mode of 4-ring cycle $\mathrm{Mg}-\mathrm{Cl}-\mathrm{Mg}-\mathrm{Cl}$ at $382 \mathrm{~cm}^{-1}$; ii) the band at $476 \mathrm{~cm}^{-1}$ for the synchronous Ti1, Ti2 pyramidalization; iii) the band at $495 \mathrm{~cm}^{-1}$ mainly comes from the 4-ring stretching Ti-Cl-Ti-Cl. Briefly, the Raman signal of dimeric adduct $\mathrm{Ti}_{2} \mathrm{Cl}_{8}$ on $\mathrm{P}$ sites when compared to monomeric $\mathrm{TiCl}_{4}$ adsorbed on T sites, shows that: i) the close bands at 377 and $385 \mathrm{~cm}^{-1}$ related to $\mathrm{P} \mathrm{Mg}^{2+}$ sites, are partly reduced (mostly the first one) and the two bands at 387 and $395 \mathrm{~cm}^{-1}$ referring to deformation of four-membered Ti-Cl-Ti-Cl ring will rise; ii) in the region between 440 and $540 \mathrm{~cm}^{-1}$ we identify a triplet with a very weak signal at $458 \mathrm{~cm}^{-1}$, the most intense band at $476 \mathrm{~cm}^{-1}$ (shifted by $11 \mathrm{~cm}^{-1}$ with respect to the monomeric case), the less intense band at $495 \mathrm{~cm}^{-1}$ (redshifted by $10 \mathrm{~cm}^{-1}$ from the monomeric case). The Raman features of the two adducts are different in shapes and show a significant difference in frequencies.

1 D’Amore, M.; Credendino, R.; Budzelaar, P. H. M.; Causá, M.; Busico, V. A Periodic Hybrid DFT Approach (Including Dispersion) to $\mathrm{MgCl}_{2}$-supported Ziegler-Natta catalysts 1: $\mathrm{TiCl}_{4}$ Adsorption on $\mathrm{MgCl}_{2}$ Crystal Surfaces. J. Catal. 2012, 286, 103-110.

2 Becke, A. D. A Multicenter Numerical Integration Scheme for Polyatomic Molecules. J. Chem. Phys. 1988, 88, 2547-2553.

3 Pascale, F.; Zicovich-Wilson, C. M.; Gejo, F. L.; Civalleri, B.; Orlando, R.; Dovesi, R. The Calculation of the Vibrational Frequencies of the Crystalline Compounds and its Implementation in the CRYSTAL Code. J. Comput. Chem. 2004, 25, 888-897.

4 Zicovich-Wilson, C. M.; Pascale, F.; Roetti, C.; Saunders, V. R.; Orlando, R.; Dovesi, R. Calculation of the Vibration Frequencies of $\alpha$-Quartz: The Effect of Hamiltonian and Basis Set. J. Comput. Chem. 2004, 25, 1873-1881. 
5 Erba, A.; Ferrabone, M.; Orlando, R.; Dovesi, R. Accurate Dynamical Structure Factors from Ab Initio Lattice Dynamics: The case of Crystalline Silicon. J. Comput. Chem. 2013, 34, $346-354$.

${ }^{6}$ Carteret, C.; De La Pierre, M.; Dossot, M.; Pascale, F.; Erba, A.; Dovesi, R. The Vibrational Spectrum of $\mathrm{CaCO}_{3}$ Aragonite: a Combined Experimental and Quantum-Mechanical Investigation. J. Chem. Phys. 2013, 138, 014201.

7 Baima, J.; Ferrabone, M.; Orlando, R.; Erba, A.; Dovesi, R. Thermodynamics and Phonon Dispersion of Pyrope and Grossular Silicate Garnets from Ab Initio Simulations. Phys. Chem. Minerals 2016, 43, 137-149.

8 Dall'Olio, S.; Dovesi, R.; Resta, R. Spontaneous polarization as a Berry phase of the HartreeFock wave function: The case of $\mathrm{KNbO}_{3}$. Phys. Rev. B 1997, 56, 10105-10114.

9 Maschio, L.; Kirtman, B.; Rérat, M.; Orlando, R.; Dovesi, R. Ab initio Analytical Raman Intensities for Periodic Systems Through a Coupled Perturbed Hartree-Fock/Kohn-Sham Method in an Atomic Orbital Basis. I. Theory. J. Chem. Phys. 2013, 139, 164101.

10 Maschio, L.; Kirtman, B.; Rérat, M.; Orlando, R.; Dovesi, R. Ab Initio Analytical Raman Intensities for Periodic Systems Through a Coupled Perturbed Hartree-Fock/Kohn-Sham Method in an Atomic Orbital Basis. II. Validation and Comparison with Experiments. J. Chem. Phys. 2013, 139, 164102. 
Tables 
Table S1: B3LYP-D2, M06 and PBE0-D3 adsorption energies in $(\mathrm{kJ} / \mathrm{mol})$ for the adducts $\mathrm{TiCl}_{4}$ on site $\mathrm{B}$ and $\mathrm{E}$ and $\mathrm{Ti}_{2} \mathrm{Cl}_{8}$ dimer on site $\mathrm{E}$ of Figure 2.

\begin{tabular}{c|c|cccc}
\hline & & \multicolumn{4}{|c}{$\Delta E_{a d s}$} \\
\hline Site & adduct & B3LYP-D2 & M06 & PBE0-D3 & B3LYP-D2 $2_{\text {surf }}$ \\
\hline B & $\mathrm{TiCl}_{4} / \mathrm{MgCl}_{2}$ & -38.6 & -68.8 & -90.6 & -64.8 \\
E & $\mathrm{TiCl}_{4} / \mathrm{MgCl}_{2}$ & -37.1 & -45.4 & -54.7 & -31.0 \\
E & $\mathrm{Ti}_{2} \mathrm{Cl}_{8} / \mathrm{MgCl}_{2}$ & -19.8 & -49.8 & -67.3 & -5.86 \\
\hline \hline
\end{tabular}

